To the Editors

\title{
A rare and unusual case of Kawasaki syndrome in a 2.5 months old baby
}

\author{
Sri Lanka Journal of Child Health, 2001; 30: 80-1
}

(Key words: Kawasaki syndrome)

Kawasaki syndrome (KS) is an acute febrile illness associated with multiorgan vasculitis. It is widely believed to be of infectious aetiology. However, no specific infectious agent has been consistently isolated from patients with this disease. It was first described in Japan by Tomisaku Kawasaki ${ }^{1}$ in 1967 and subsequently recognized world wide in children of every racial group. The Sri Lanka Journal of Child Health had a feature on the disease recently $y^{2}$ I wish to report the disease in a 2.5 months old baby.

A $2 \frac{1}{2}$ month old female infant presented with a 4 day history of high fever. The mother reported that the infant had no feeding difficulty and her cry was normal. She had been treated only with antipyretics prior to admission. She is the first child of a healthy 41 year old lady. The baby was born at term with a birth weight of $3.2 \mathrm{~kg}$ after an uncomplicated pregnancy and remained well till the present admission. Initial physical examination revealed a febrile ill infant with 2 erythematous plaques over her legs. Each was $0.5 \mathrm{~cm}$ in diameter. The rest of the examination was normal.

After a septic screen she was started on IV penicillin and cefotaxime for a possible septicaemia. The white blood cell count was $12.9 \times 10^{9} / \mathrm{L}$ with $89 \%$ polymorphonuclear leukocytes and the platelet count was $393 \times 10^{9} / \mathrm{L}$. ESR was $74 \mathrm{~mm} / \mathrm{1}^{\text {st }}$ hour while the CRP was $21.62 \mathrm{mg} / \mathrm{dl}$. CSF examination did not reveal any abnormality. Blood culture remained sterile.

On day 4 of IV antibiotics she continued to be febrile and ill. On further analysis she was noted to have mild redness of bulbar conjunctivae and lips. The possibility of atypical Kawasaki disease was raised and further investigations were carried out. EGG, liver function tests, baseline echocardiography and platelet counts were all normal.

She was treated with $2 \mathrm{gm} / \mathrm{kg}$ IV immunoglobulin and aspirin $(100 \mathrm{mg} / \mathrm{kg} /$ day in four equal doses) on clinical suspicion. Within 24 hours her fever subsided and her clinical condition improved. She was discharged home on aspirin. On the 14th day of illness she developed periungual desquamation and the platelet count was $750 \times 10^{9} / \mathrm{L}$. A repeat echocardiogram in the 3rd week of illness demonstrated no cardiac complications.

Kawasaki syndrome has a variety of clinical manifestations, which are shared with other infections and reactive disorders. This can result in diagnostic dilemmas, particularly in atypical cases. This $21 / 2$ month old infant who was ill with unexplained fever and erythematous rash was initially treated as for septicaemia. Continued fever despite broad-spectrum intravenous antibiotics and the presence of a sterile blood culture in an infant who had not received antibiotics prior to admission made us consider an alternative diagnosis. Conjunctival injection and mild erythema of lips which appeared later supported our diagnosis. Periungual desquamation, which was noticed about the 14lh day of illness, established the diagnosis. (Figure 1)

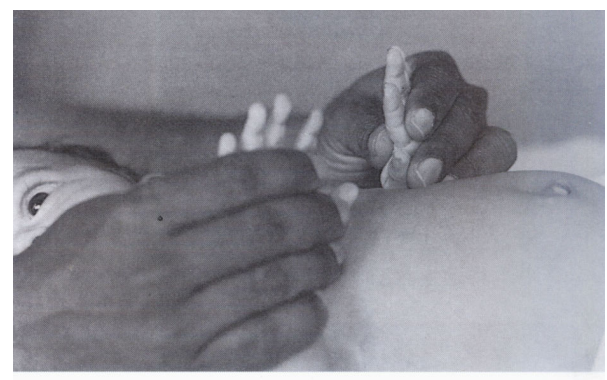

Figure 1. Fingers showing periungual desquamatio

Myocarditis in Kawasaki syndrome occurs during the first week after onset of fever and is often associated with pericardial effusion. Coronary artery abnormalities occur in nearly $25 \%$ of Kawasaki syndrome patients not treated with IVIG within 10 days of onset of fever. This is particularly so for children under 6 months of age. Studies of immune response in patients with Kawasaki syndrome reveal an unusual degree of stimulation and it is likely that IVIG modifies the inflammatory response through more than one mechanism. As there is no laboratory investigation to confirm a definitive diagnosis it is a challenge to recognize atypical cases of the syndrome.

\section{References}

1. Kawasaki T, Kosaki F, Okawas et al. A new infantile acute febrile mucocutaneous lymph node syndrome prevailing in Japan. Paediatrics 1974; 54: 271-6.

2. Fernando T A. Kawasaki Syndrome. Sri Lanka Journal of Child Health 2000; 29(1): 29-30.

P M G. Punchihewa, Consultant Paediatrician, Teaching Hospital, Galle.

L P C Saman Kumara, Registrar, Teaching Hospital, Galle. 
